\title{
N-Heterocyclic Carbene Catalyzed Enantioselective Annulation of Benzothiazolyl Ethyl Acetates with 2-Bromoenals
}

\author{
Qijian Ni \\ Jiawen Xiong \\ Xiaoxiao Song \\ Gerhard Raabe \\ Dieter Enders*
}

Institute of Organic Chemistry, RWTH Aachen University, Landoltweg 1, 52074 Aachen, Germany

enders@rwth-aachen.de

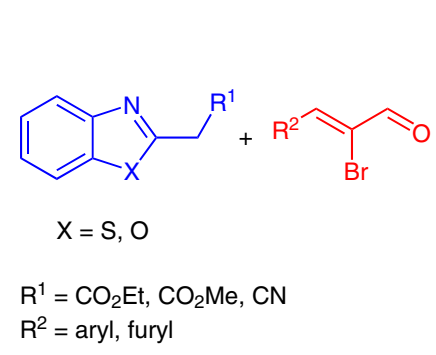

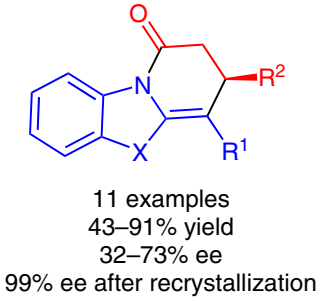

precatalyst (10 mol\%)

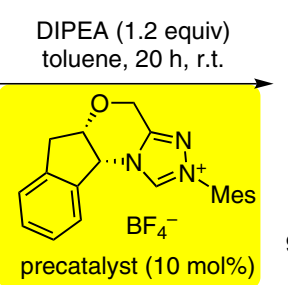

$99 \%$ ee after recrystallization
Received: 30.03.2015

Accepted after revision: 16.05.2015

Published online: 11.06 .2015

DOI: 10.1055/s-0034-1381004; Art ID: st-2015-b0228-I

License terms: (C) (1)

Abstract An N-heterocyclic carbene catalyzed enantioselective [3+3] annulation of benzothiazolyl acetates with 2-bromoenals has been developed. The protocol provides a direct asymmetric synthesis of dihydro- $1 \mathrm{H}$-benzothiazolopyridinones in good to very good yields and medium ee values. In many cases, the virtually enantiopure heterocycles are available through a single recrystallization ( $99 \%$ ee).

Key words asymmetric synthesis, N-heterocyclic carbene, organocatalysis, annulation, dihydrobenzothiazolopyridinones

Since the seminal reports by the groups of Glorius and Bode in $2004^{1}$ much attention has been paid to develop novel $\mathrm{N}$-heterocyclic carbene (NHC)-catalyzed cyclization/annulation methods. ${ }^{2}$ Especially NHC-based $\alpha, \beta$-unsaturated acylazolium intermediates turned out to be excellent electrophiles, ${ }^{3}$ which could undergo stepwise Michael-acylation or sigmatropic rearrangement-acylation reactions with a variety of dinucleophiles such as 1,3-diketones, ${ }^{4}$ enamines, ${ }^{5}$ naphthols, ${ }^{6}$ or enolizable aldehydes. ${ }^{7}$ Recently, the Ye group reported a [3+3] cyclocondensation of bromoenals with ketimines in the asymmetric synthesis of dihydropyridinones. ${ }^{8}$ Very recently our group developed NHC-catalyzed enantioselective annulations of indolin-3ones with 2-bromoenals to form dihydropyranoindolones. ${ }^{9}$ In view of the importance of such heterocycles as potentially bioactive compounds the research for further suitable nucleophiles in these annulation protocols is highly desirable.

The dihydro- $1 H$-benzothiazolopyridine core is present in various biologically active natural products and has found widespread applications in numerous pharmaceuticals, such as antitumor ${ }^{10}$ and antibacterial drugs. ${ }^{11}$ Howev- er, only a few asymmetric syntheses have been investigated. In 2013 Smith and co-workers reported an asymmetric annulation of benzothiazolyl ketones with $\alpha, \beta$-unsaturated anhydrides catalyzed by the isothiourea HBTM 2.1 (Scheme 1, a). ${ }^{12}$ Very recently, we reported a Mannich-lactamization domino reaction of $\mathrm{N}$-(benzothiazolyl)imines with 2-chloroaldehydes for the synthesis of benzothiazolo-pyrimidinones (Scheme 1, b) ${ }^{13}$, followed by an extended work on 1azadiene-Diels-Alder reactions of styrylbenzo[d]thiazoles with $\alpha$-chloroaldehydes (Scheme 1, c). ${ }^{14}$ Herein, we report the asymmetric synthesis of dihydro- $1 H$-benzothiazolopyridine-2-ones via the formal [3+3] annulation reaction of 2bromoenals with 2 -substituted benzo[d]thiazoles (Scheme $1, d)$.

Initially, we performed the model reaction of 2-(benzothiazol-2-yl) ethyl acetate (1a) with 2-bromocinnamaldehyde (2a) at room temperature in toluene in the presence of $\mathrm{N}, \mathrm{N}$-diisopropyl ethylamine (DIPEA) and $10 \mathrm{~mol} \%$ of the triazolium precatalyst $\mathbf{A}$, which proceeded smoothly and gave a $45 \%$ yield of the product 3a (Table 1, entry 1 ). Chiral triazolium salts B-F were also screened and a good yield of $83 \%$ and an enantiomeric excess of $80 \%$ were obtained with the triazolium salt $\mathbf{C}$ (Table 1, entry 3 ). Next we screened a series of bases, however, organic bases such as DABCO, TMEDA, TBD, or DBU and inorganic bases such as $\mathrm{K}_{3} \mathrm{PO}_{4}$ and $\mathrm{K}_{2} \mathrm{CO}_{3}$ gave inferior results (Table 1, entries 7-12). We then tested a series of solvents in the presence of precatalyst $\mathbf{C}$ and DIPEA at room temperature. Unfortunately, no improvement was obtained (Table 1, entries 13-17), even with the mixed solvents of toluene-THF (Table 1, entry 18) and toluene-MeCN (Table 1, entry 19). Inspired by recent reports on the NHC-Lewis acid strategy ${ }^{15}$ we examined some Lewis acids as additives in our protocol. The strong Lewis acid $\mathrm{Sc}(\mathrm{OTf})_{3}$ lowered the reactivity and enantioselectivity (Table 1, entry 20), and the use of the weak Lewis acid LiCl even inversed the asymmetric induction (Table 1 , entry 21 ). 
previous reports:

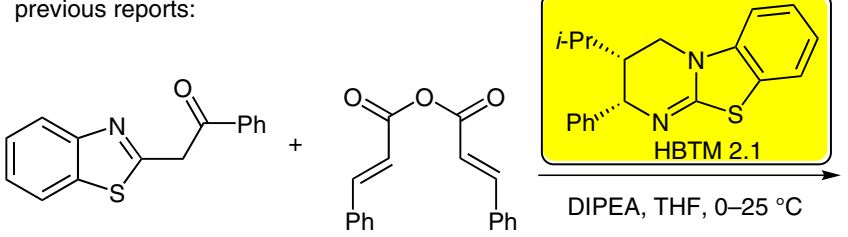<smiles>O=C(C1=C2Sc3ccccc3N2C(=O)C[C@@H]1c1ccccc1)c1ccccc1</smiles>

$86 \%$ yield, $83 \%$ ee

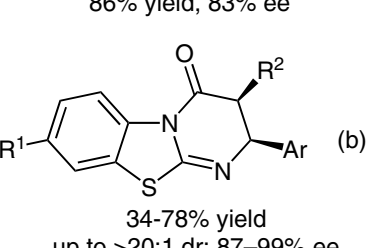<smiles>[123I]/C=N/c1nc2ccc([Tl])cc2s1</smiles><smiles>[R]C(Cl)C=O</smiles>

DABCO (2.2 equiv)

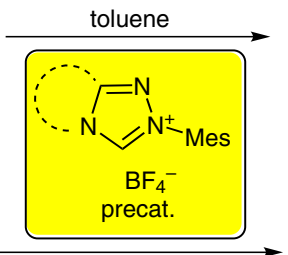<smiles>[R]C=C(C#N)c1[X]c2ccc([R])cc2n1</smiles><smiles>[R]C(Cl)C=O</smiles>

DIPEA, r.t., $16 \mathrm{~h}$ toluene-MeCN (1:2)

$$
\mathrm{X}=\mathrm{S}, \mathrm{O}
$$

this work:<smiles>[R]C=C(Br)C=O</smiles>

NHC catalyst

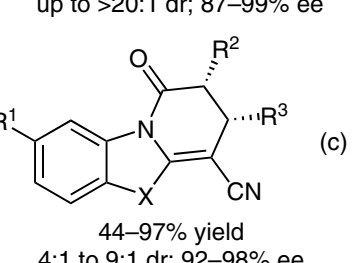<smiles>[X]c1ccccc1N1C=C(C(=O)OCC)C([R])CC1=O</smiles>

Finally, we lowered the reaction temperature, however, no further improvement on enantioselectivity was obtained at $5{ }^{\circ} \mathrm{C}$ (Table 1 , entry 22 ) and $-20^{\circ} \mathrm{C}$ (Table 1 , entry 23 ).

We then amplified the scale of the model reaction to 0.5 mmol, which afforded $3 a$ in $77 \%$ yield and $65 \%$ ee. ${ }^{16}$ Fortunately, a single recrystallization allowed to access the virtually enantiopure product ( $99 \%$ ee, Table 2 , entry 1 ). Next we investigated the substrate scope of this protocol by variation of the 2-substituted benzo[d]thiazole component $\mathbf{1}$. A methyl ester and a cyano group as $\mathrm{R}^{1}$ gave the desired adducts in good to excellent yields and moderate ee values (Table 2, entries 2 and 3). Gratifyingly, 2-(benzoxazol-2yl)acetonitrile underwent the transformation smoothly and furnished the desired [3+3] annulation product in moderate yield and ee (Table 2, entry 4). Furthermore, various electron-donating and electron-withdrawing groups, as well as ortho substituents attached to the aryl group of the bromoenals $\left(R^{2}\right)$ were well tolerated, leading to the desired products in good yields and moderate enantiomeric excess (Table 2, entries 5-10). Notably, several products could be obtained as virtually enantiopure compounds ( $99 \%$ ee) after a single recrystallization. Additionally, a heterocyclic 2-furyl substituent $R^{2}$ can be used resulting in a $77 \%$ yield and $58 \%$ ee (Table 2 , entry 11 ).

The absolute configuration was unambiguously determined to be $S$ by X-ray crystal-structure analysis of the methyl acetate $\mathbf{3 b}$ (Figure 1). ${ }^{17}$
A plausible reaction mechanism for this NHC-catalyzed formal [3+3] annulation is shown in Scheme 2. The addition of the NHC $\mathbf{C}^{\prime}$ to the 2-bromoenal 2 leads to the Breslow intermediate $\mathbf{I}$, also drawn as its mesomeric zwitterionic form. After tautomerization to II, the subsequent loss of bromide generates the $\alpha, \beta$-unsaturated acylazolium key intermediate III. The base-mediated Michael addition of the benzothiazolyl ethyl acetates $\mathbf{1}$ affords the adduct $\mathbf{I V}$, followed by proton transfer and lactamization via $\mathbf{V}$ to furnish the final product 3 and to return the NHC catalyst.

In summary, we have developed a novel NHC-catalyzed asymmetric annulation of 2-(benzothiazol-2-yl) acetates with 2-bromoenals. The protocol tolerates quite a range of substrates including a benzoxazolyl acetonitrile and give

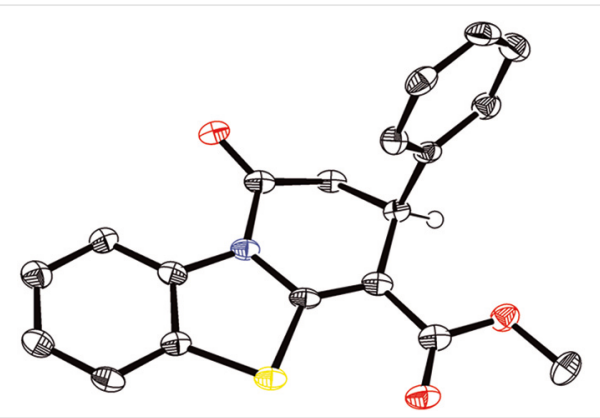

Figure 1 Absolute configuration [X-ray, $\mathrm{X}_{\mathrm{abs}}=0.078$ (32)] of $\mathbf{3 b}$ 
Table 1 Optimization of the Reaction Conditions ${ }^{\mathrm{a}}$

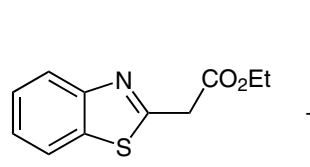

$1 a$<smiles>O=C/C(Br)=C/c1ccccc1</smiles>

2a

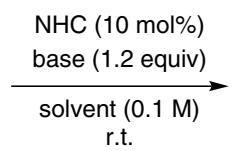

r.t.<smiles>CCOC(=O)C1=C2Sc3ccccc3N2C(=O)C[C@@H]1c1ccccc1</smiles>

$3 a$<smiles>c1ccc(-[n+]2cn3c(n2)CCC3)cc1</smiles>

A<smiles>FC(F)(F)c1cn2c(n1)CC[C@@H]2Cc1ccccc1</smiles><smiles>[X][n+]1cn2c(n1)CO[C@H]1Cc3ccccc3[C@@H]12</smiles>

C $\mathrm{X}=\mathrm{BF}_{4}, \mathrm{R}=\mathrm{Mes}$

D $\mathrm{X}=\mathrm{BF}_{4}, \mathrm{R}=\mathrm{C}_{6} \mathrm{~F}_{5}$

\begin{tabular}{|c|c|c|c|c|c|c|}
\hline Entry & $\mathrm{NHC}$ & Solvent & Base & Additive & Yield (\%) & ee $(\%)^{c}$ \\
\hline 1 & A & toluene & DIPEA & - & 45 & - \\
\hline 2 & B & toluene & DIPEA & - & 29 & -26 \\
\hline 3 & C & toluene & DIPEA & - & 83 & 80 \\
\hline 4 & D & toluene & DIPEA & - & 61 & 75 \\
\hline 5 & $E$ & toluene & DIPEA & - & 71 & 1 \\
\hline 6 & $\mathbf{F}$ & toluene & DIPEA & - & n.r. & - \\
\hline 7 & C & toluene & DABCO & - & 66 & 79 \\
\hline 8 & C & toluene & TMEDA & - & 86 & 68 \\
\hline 9 & $\mathrm{C}$ & toluene & $\mathrm{TBD}^{\mathrm{d}}$ & - & trace & - \\
\hline 10 & C & toluene & DBU & - & 23 & -22 \\
\hline 11 & C & toluene & $\mathrm{K}_{3} \mathrm{PO}_{4}$ & - & 20 & 63 \\
\hline 12 & C & toluene & $\mathrm{K}_{2} \mathrm{CO}_{3}$ & - & 9 & 68 \\
\hline 13 & C & $\mathrm{MeCN}$ & DIPEA & - & 80 & 73 \\
\hline 14 & C & $\mathrm{CH}_{2} \mathrm{Cl}_{2}$ & DIPEA & - & 80 & 32 \\
\hline 15 & C & THF & DIPEA & - & 26 & 41 \\
\hline 16 & C & MTBE & DIPEA & - & 57 & 73 \\
\hline 17 & C & mesitylene & DIPEA & - & 46 & 82 \\
\hline 18 & C & toluene-THF (10:1) & DIPEA & - & 70 & 79 \\
\hline 19 & C & toluene-MeCN (10:1) & DIPEA & - & 82 & 69 \\
\hline 20 & C & toluene & DIPEA & $\mathrm{Sc}(\mathrm{OTf})_{3}$ & 29 & 66 \\
\hline 21 & C & toluene & DIPEA & $\mathrm{LiCl}$ (1 equiv) & 29 & -18 \\
\hline $22^{e}$ & C & toluene & DIPEA & - & 76 & 76 \\
\hline $23^{f}$ & C & toluene & DIPEA & - & 76 & 77 \\
\hline
\end{tabular}

a Reaction conditions: 1a $(0.2 \mathrm{mmol}), 2 \mathrm{a}(0.3 \mathrm{mmol})$, precatalyst $(0.02 \mathrm{mmol})$, base $(0.24 \mathrm{mmol})$, solvent $(2 \mathrm{~mL})$, r.t., under argon, $20 \mathrm{~h}$.

b Yield of isolated product $3 a$ after column chromatography.

c The ee was determined by HPLC on a chiral stationary phase.

${ }^{\mathrm{d}} \mathrm{TBD}=$ 1,5,7-triazabicyclo[4.4.0]dec-5-ene.

e Performed at $5{ }^{\circ} \mathrm{C}$ for $4 \mathrm{~d}$.

${ }^{f}$ Performed at $-20^{\circ} \mathrm{C}$ for $4 \mathrm{~d}$.

rise to the corresponding dihydro- $1 H$-benzothiazolopyridinones in moderate to very good yields and medium ee values. However, in several cases virtually enantiopure products $(99 \%$ ee) could be obtained via a single recrystallization.

\section{Acknowledgment}

We thank the European Research Council (ERC Advanced Grant 320493 'DOMINOCAT') for financial support. Q.N. and X.S. are grateful to the China Scholarship Council for a fellowship. 
Table 2 Substrate Scope

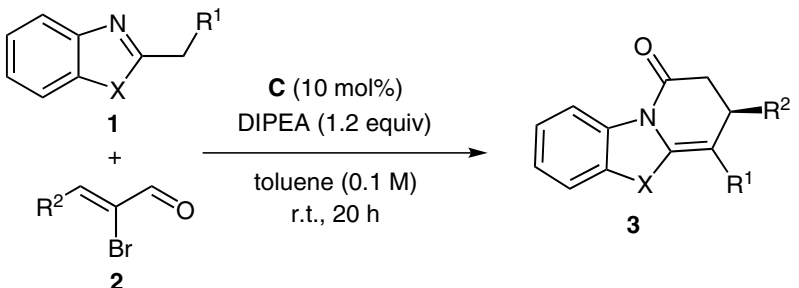

\begin{tabular}{|c|c|c|c|c|c|c|}
\hline Entry & 3 & $\mathrm{R}^{1}$ & $R^{2}$ & $x$ & Yield (\%) ${ }^{\mathrm{b}}$ & ee $(\%)^{c, d}$ \\
\hline 1 & $3 a$ & $\mathrm{CO}_{2} \mathrm{Et}$ & $\mathrm{Ph}$ & $\mathrm{S}$ & 77 & 65 (99) \\
\hline 2 & $3 b$ & $\mathrm{CO}_{2} \mathrm{Me}$ & $\mathrm{Ph}$ & $S$ & 91 & 64 \\
\hline 3 & $3 c$ & $\mathrm{CN}$ & $\mathrm{Ph}$ & $S$ & 74 & 32 \\
\hline 4 & $3 d$ & $\mathrm{CN}$ & $\mathrm{Ph}$ & $\mathrm{O}$ & 43 & 55 \\
\hline 5 & $3 e$ & $\mathrm{CO}_{2} \mathrm{Et}$ & 4- $\mathrm{MeC}_{6} \mathrm{H}_{4}$ & $S$ & 64 & 68 \\
\hline 6 & $3 f$ & $\mathrm{CO}_{2} \mathrm{Et}$ & $4-\mathrm{MeOC}_{6} \mathrm{H}_{4}$ & $S$ & 69 & $62(99)$ \\
\hline 7 & $3 g$ & $\mathrm{CO}_{2} \mathrm{Et}$ & 2-MeO-5- $\mathrm{BrC}_{6} \mathrm{H}_{3}$ & $S$ & 80 & $65(92)$ \\
\hline 8 & $3 \mathrm{~h}$ & $\mathrm{CO}_{2} \mathrm{Et}$ & $2-\mathrm{MeOC}_{6} \mathrm{H}_{4}$ & $S$ & 86 & 66 (99) \\
\hline 9 & $3 \mathbf{i}$ & $\mathrm{CO}_{2} \mathrm{Et}$ & $4-\mathrm{ClC}_{6} \mathrm{H}_{4}$ & $\mathrm{~S}$ & 72 & 73 \\
\hline 10 & $3 \mathbf{j}$ & $\mathrm{CO}_{2} \mathrm{Et}$ & $4-\mathrm{BrC}_{6} \mathrm{H}_{4}$ & $S$ & 83 & 70 (99) \\
\hline 11 & $3 k$ & $\mathrm{CO}_{2} \mathrm{Et}$ & 2-furyl & $S$ & 77 & 58 \\
\hline
\end{tabular}

${ }^{a}$ Reaction conditions: $1(0.5 \mathrm{mmol}), \mathbf{2}(0.75 \mathrm{mmol})$, precatalyst C $(0.05 \mathrm{mmol})$, DIPEA $(0.6 \mathrm{mmol})$, toluene $(5 \mathrm{~mL})$, r.t., under argon, $20 \mathrm{~h}$.

${ }^{\mathrm{b}}$ Yield of isolated product $\mathbf{3}$ after column chromatography.

' The ee was determined by HPLC on a chiral stationary phase.

${ }^{d}$ The value in parentheses refers to the ee after recrystallization.

\section{Supporting Information}

Supporting information for this article is available online at http://dx.doi.org/10.1055/s-0034-1381004.

\section{References and Notes}

(1) (a) Burstein, C.; Glorius, F. Angew. Chem. Int. Ed. 2004, 43, 6205. (b) Sohn, S. S.; Rosen, E. L.; Bode, J. W. J. Am. Chem. Soc. 2004, 126, 14370. (c) For a review, see: Nair, V.; Menon, R. S.; Biju, A. T.; Sinu, C. R.; Paul, R. R.; Jose, A.; Sreekumar, V. Chem. Soc. Rev. 2011, 40, 5336.

(2) For selected reviews on NHC organocatalysis, see: (a) Enders, D.; Niemeier, O.; Henseler, A. Chem. Rev. 2007, 107, 5606. (b) Nair, V.; Vellalath, S.; Babu, B. P. Chem. Soc. Rev. 2008, 37, 2691. (c) Biju, A. T.; Kuhl, N.; Glorius, F. Acc. Chem. Res. 2011, 44, 1182. (d) Grossmann, A.; Enders, D. Angew. Chem. Int. Ed. 2012, 51, 314. (e) Vora, H. U.; Wheeler, P.; Rovis, T. Adv. Synth. Catal. 2012, 354, 1617. (f) Bugaut, X.; Glorius, F. Chem. Soc. Rev. 2012, 41, 3511. (g) Izquierdo, J.; Hutson, G. E.; Cohen, D. T.; Scheidt, K. A. Angew. Chem. Int. Ed. 2012, 51, 11686. (h) Ryan, S. J.; Candish, L.; Lupton, D. W. Chem. Soc. Rev. 2013, 42, 4906. (i) Hopkinson, M. N.; Richter, C.; Schedler, M.; Glorius, F. Nature (London, U.K.) 2014, 510, 485.

(3) For selected examples for the generation of $\alpha, \beta$-unsaturated acylazolium intermediates, see: (a) Ryan, S. J.; Candish, L.; Lupton, D. W. J. Am. Chem. Soc. 2009, 131, 14176. (b) De Sarkar, S.; Studer, A. Angew. Chem. Int. Ed. 2010, 49, 9266. (c) Kaeobamrung, J.; Mahatthananchai, J.; Zheng, P.; Bode, J. W. J. Am. Chem. Soc. 2010, 132, 8810. (d) Yao, C.; Wang, D.; Lu, J.; Li, T.; Jiao, W.; Yu, C. Chem. Eur. J. 2012, 18, 1914. (e) Cheng, J.; Huang, Z.; Chi, Y. R. Angew. Chem. Int. Ed. 2013, 52, 8592. (f) Chen, X.-Y.; Gao, Z.-H.; Song, C.-Y.; Zhang, C.-L.; Wang, Z.-X.; Ye, S. Angew. Chem. Int. Ed. 2014, 53, 11611.

(4) (a) Rong, Z.-Q.; Jia, M.-Q.; You, S.-L. Org. Lett. 2011, 13, 4080. (b) Sun, F.-G.; Sun, L.-H.; Ye, S. Adv. Synth. Catal. 2011, 353, 3134.

(5) (a) Wanner, B.; Mahatthananchai, J.; Bode, J. W. Org. Lett. 2011, 13, 5378. (b) Yetra, S. R.; Bhunia, A.; Patra, A.; Mane, M. V.; Vanka, K.; Biju, A. T. Adv. Synth. Catal. 2013, 355, 1089.

(6) Mahatthananchai, J.; Kaeobamrung, J.; Bode, J. W. ACS Catal. 2012, 2, 494.

(7) Yetra, S. R.; Kaicharla, T.; Kunte, S. S.; Gonnade, R. G.; Biju, A. T. Org. Lett. 2013, 15, 5202.

(8) Zhang, H.-M.; Jia, W.-Q.; Liang, Z.-Q.; Ye, S. Asian J. Org. Chem. 2014, 3, 462.

(9) Ni, Q.; Song, X.; Raabe, G.; Enders, D. Chem. Asian J. 2014, 9, 1535; and references cited therein.

(10) Haddach, M.; Schwaebe, M. K.; Michaux, J.; Nagasawa, J.; O’Brien, S. E.; Whitten, J. P.; Pierre, F.; Kerdoncuff, P.; Darjania, L.; Stansfield, R.; Drygin, D.; Anderes, K.; Proffitt, C.; Bliesath, J.; Siddiqui-Jain, A.; Omori, M.; Huser, N.; Rice, W. G.; Ryckman, D. M. ACS Med. Chem. Lett. 2012, 3, 602.

(11) Dinakaran, M.; Senthilkumar, P.; Yogeeswari, P.; China, A.; Nagaraja, V.; Sriram, D. Bioorg. Med. Chem. 2008, 16, 3408. 


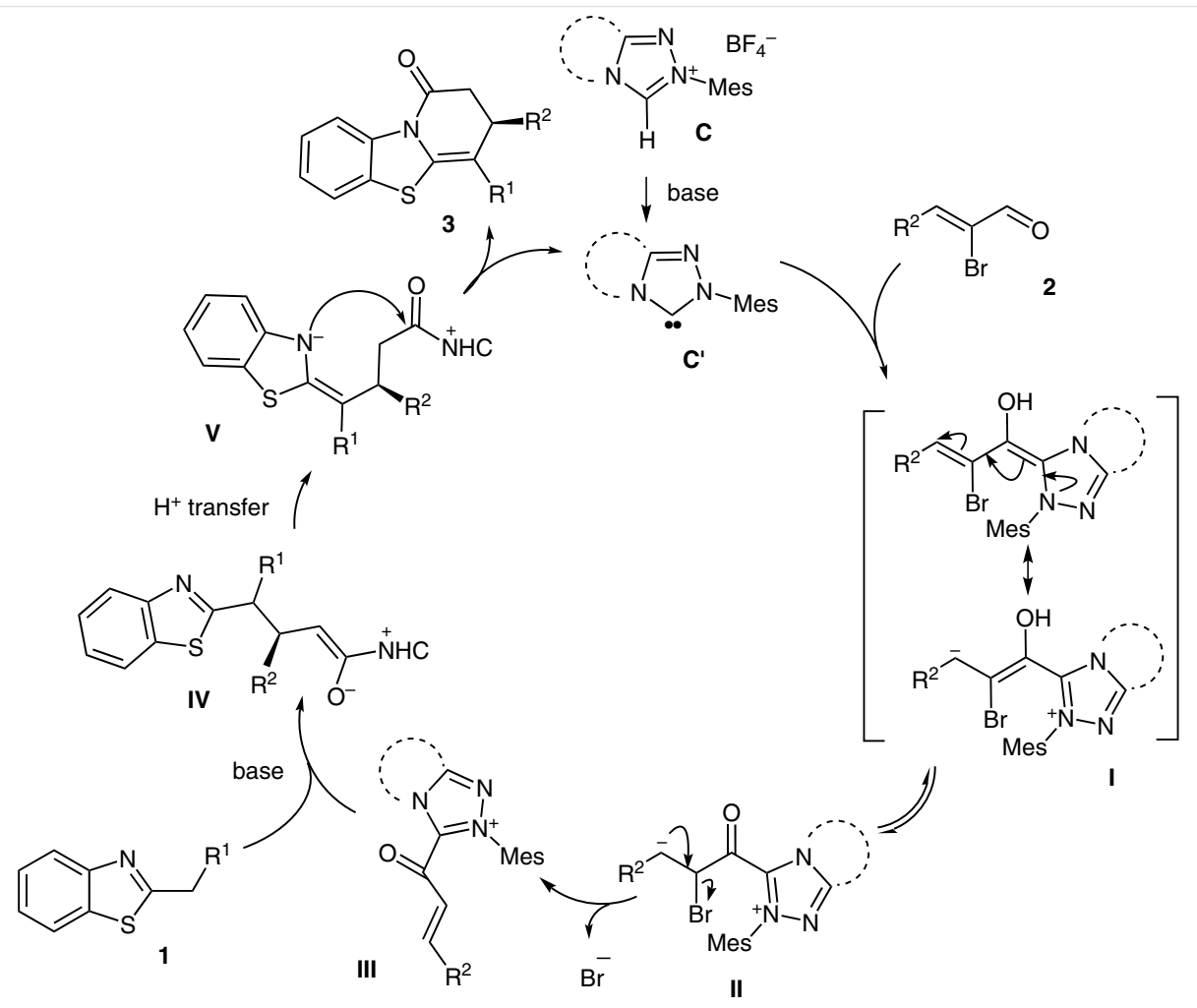

Scheme 2 Proposed reaction pathway

(12) Robinson, E. R. T.; Fallan, C.; Simal, C.; Slawin, A. M. Z.; Smith, A. D. Chem. Sci. 2013, 4, 2193.

(13) Ni, Q.; Song, X.; Xiong, J.; Raabe, G.; Enders, D. Chem. Commun. 2015, 51, 1263 .

(14) Song, X.; Ni, Q.; Chen, Z.; Raabe, G.; Enders, D. Synthesis 2015 , $47,421$.

(15) (a) Raup, D. E. A.; Cardinal-David, B.; Holte, D.; Scheidt, K. A. Nat. Chem. 2010, 2, 766. (b) Cohen, D. T.; Cardinal-David, B.; Scheidt, K. A. Angew. Chem. Int. Ed. 2011, 50, 1678. (c) DugalTessier, J.; O’Bryan, E. A.; Schroeder, T. B. H.; Cohen, D. T.; Scheidt, K. A. Angew. Chem. Int. Ed. 2012, 51, 4963.

(16) General Procedure for the Synthesis of Dihydro-1H-benzothiazolopyridinones $3 a-k$

To an oven-dried and argon-filled Schlenk tube was added 2substituted benzo[d]thiazole component $1(0.5 \mathrm{mmol}), 2$-bromoenal 2 ( $0.75 \mathrm{mmol}, 1.5$ equiv), triazolium salt C $(0.05 \mathrm{mmol}$, $10 \mathrm{~mol} \%$ ), and DIPEA (0.6 mmol, 1.2 equiv) in toluene ( $5 \mathrm{~mL})$. The mixture was stirred at r.t. and monitored by TLC until completion of the reaction. The residue was purified by flash chromatography on silica gel [ $n$-pentane- $\mathrm{Et}_{2} \mathrm{O}(10: 1)$ or $n$-pentane$\mathrm{CH}_{2} \mathrm{Cl}_{2}$ (1:1 to $\left.\left.1: 2\right)\right]$ to afford the products $3 \mathbf{a}-\mathbf{k}$ as orange or yellow solids.
Ethyl (S)-1-0xo-3-phenyl-2,3-dihydro-1H-benzo[4,5] thiazolo[3,2-a]pyridine-4-carboxylate (3a)

Yield: $135.6 \mathrm{mg}(77 \%), \mathrm{mp} 125-127^{\circ} \mathrm{C}$. The ee $(65 \%, 99 \%$ after recrystallization) was measured by HPLC using a chiral stationary phase [Daicel IC, $n$-heptane-EtOH $=7: 3,0.7 \mathrm{~mL} / \mathrm{min}$ ), $t_{\mathrm{R}}=$ 4.53 min (major), $5.35 \mathrm{~min}$ (minor)]. $[\alpha]_{D}^{23}=+236.9$ (c 1.0, $\left.\mathrm{CHCl}_{3}\right) .{ }^{1} \mathrm{H}$ NMR $\left(600 \mathrm{MHz}, \mathrm{CDCl}_{3}\right): \delta=8.44(\mathrm{~d}, J=8.4 \mathrm{~Hz}, 1 \mathrm{H})$, 7.45 (dd, $J=7.2,1.2 \mathrm{~Hz}, 1 \mathrm{H}), 7.30-7.18(\mathrm{~m}, 7 \mathrm{H}), 4.33-4.32(\mathrm{~m}, 1$ H), 4.28-4.16 (m, $2 \mathrm{H}), 3.24$ (dd, $J=16.2,8.4 \mathrm{~Hz}, 1 \mathrm{H}$ ), 3.02 (dd, $J$ $=16.2,1.8 \mathrm{~Hz}, 1 \mathrm{H}), 1.23(\mathrm{t}, J=7.2 \mathrm{~Hz}, 3 \mathrm{H}) .{ }^{13} \mathrm{C}$ NMR $(150 \mathrm{MHz}$, $\left.\mathrm{CDCl}_{3}\right): \delta=168.2,166.6,152.3,141.4,136.8,128.9(2 \mathrm{C}), 127.2$, 127.0, 126.5 (2 C), 126.5, 125.5, 121.4, 117.4, 100.4, 60.7, 40.1, 36.8, 14.3. MS (EI, $70 \mathrm{eV}): m / z(\%)=351(100)\left[\mathrm{M}^{+}\right], 322(36)$, 278 (40), 249 (44), 236 (71), 115 (19), 77 (17). IR (ATR): 3851, $3613,3401,3060,2980,2921,2645,2325,2037,1903,1803$, 1707, 1660, 1556, 1455, 1359, 1305, 1263, 1194, 1146, 1106, 1034, 939, 906, 853, 795, 748, $697 \mathrm{~cm}^{-1}$. ESI-HRMS: $\mathrm{m} / \mathrm{z}$ calcd for $\mathrm{C}_{20} \mathrm{H}_{17} \mathrm{NO}_{3} \mathrm{~S}$ [M]+: 351.0924 ; found: 351.0933 .

(17) CCDC 1056458 contains the supplementary crystallographic data for the compound $\mathbf{3 b}$ reported in this paper. These data can be obtained free of charge from The Cambridge Crystallographic Data Centre via www.ccdc.cam.ac.uk/data_request/cif. 